\title{
EFAST NA SALA DE EMERGÊNCIA NO DIAGNÓSTICO DE PNEUMOTÓRAX: RELATO DE CASO
}

\author{
Imad Shehadeh* \\ Claudio Ricardo Capela Bogdan** \\ Marcelo da Silva Pereira Fernandes ${ }^{*+*}$ \\ Fabiane Vieira Berg Shehadeh ${ }^{* *+x}$ \\ Luiz Alfredo Calvo Fracasso ${ }^{\star * \star * x}$ \\ Lis Osaku
}

\begin{abstract}
RESUMO: A presença de ar livre no espaço pleural pode ocorrer de maneira espontânea ou adquirida e é uma condição clínica frequente no cotidiano de um pronto socorro. Vítimas de traumas torácicos podem desenvolver pneumotórax e complicações à vida. Uso de EFAST (Extended Focused Assessment with Sonography for Trauma) permitiu avanço no diagnóstico dessas lesões ameaçadoras à vida. Este relato mostra o precoce diagnóstico de pneumotórax, usando EFAST, em paciente vítima de trauma torácico fechado. Paciente masculino, 65 anos, admitido em sala de emergência de um hospital universitário, após colisão automobilística há dois dias. Apresentava dor torácica ventilatório-dependente, principalmente à esquerda, e dispneia aos grandes esforços. 0 murmúrio vesicular estava presente no hemitórax direito, mas abolido no terço inferior do hemitórax esquerdo. Submetido à $E F A S T$, que mostrou pneumotórax à esquerda. A toracostomia com drenagem pleural à esquerda foi realizada sem intercorrências e o pneumotórax foi resolvido. Recebeu alta após quatro dias de internação, com melhora clínica satisfatória e reexpansibilidade pulmonar adequada. 0 exame ultrassonográfico do tórax em vítimas de trauma tem a vantagem de ser não invasivo, de imediata avaliação e fácil portabilidade. Tem se mostrado superior ao exame de raios $-X$ de tórax, em termos de sensibilidade, e à tomografia computadorizada do tórax, quanto ao tempo de execução e exposição do paciente à radiação. Seu uso deve ser estendido e aplicado na avaliação primária de todo paciente politraumatizado atendido em sala de emergência.
\end{abstract}

PALAVRAS-CHAVE: Cirurgia; Trauma; Ultrassonografia; Pneumotórax; Emergência.

\section{EFAST IN THE EMERGENCY ROOM FOR THE DIAGNOSIS OF PNEUMOTHORAX: REPORT OF A CASE}

\begin{abstract}
Free air in the pleural space may occur spontaneously or may be acquired. It is a frequent condition in first-aid clinics. People with thorax trauma may develop pneumothorax and risky complications. The use of Extended Focused Assessment with Sonography for Trauma (EFAST) triggers progress in the diagnosis of such life-endangering lesions. Current report shows an early diagnosis of pneumothorax with the use of EFAST in a patient suffering from close thorax trauma. The 65-year-old male patient was admitted to the emergency room of a University Hospital after a two-day-old car crash. He complained of ventilator-dependent thorax pain especially on the left side and dyspnea with great efforts. Vesicular murmur was present at the right hemithorax but abolished at the third lower part of the left hemithorax. EFAST revealed left pneumothorax and a thoracostomy with left pleural drainage was performed without any inter-occurrences and the pneumothorax was solved. He quitted hospital after 4 days with a satisfactory clinical condition and adequate lung re-expandability. Ultrasonography of the thorax in trauma victims is not invasive, portable and easily executed. In fact, it proved to be better than X-ray

\footnotetext{
Médico Cirurgião do Trauma e Cirurgião Geral; Médico técnico e plantonista de cirurgia geral do Hospital Universitário de Maringá - HUM; Instrutor do ATLS, Maringá (PR), Brasil; E-mail: imadpoa@gmail.com ATLS, Maringá (PR), Brasil.
}

* Médico Cirurgião Vascular e Cirurgião Geral; Médico técnico e plantonista de cirurgia geral do Hospital Universitário de Maringá - HUM; Instrutor do

${ }^{* * *}$ Médico Cirurgião Geral, plantonista de cirurgia geral do Hospital Universitário de Maringá - HUM, (PR), Brasil.

**** Biomédica; Mestre pelo Programa de Pós-graduação em Ciências da Saúde, Universidade Estadual de Maringá - UEM, (PR), Brasil.

${ }^{*}$ Médico Cirurgião Geral, plantonista de cirurgia geral do Hospital Universitário de Maringá - HUM, (PR), Brasil.

Médica plantonista em sala de emergência da Unidade de Pronto Atendimento em Maringá (PR), Brasil.
\end{abstract}


of the thorax in terms of sensitiveness, and even better than computer tomography regarding time and exposure of the patient to radiation. Its use should be expanded and applied as a primary assessment of all polytraumatized patients attended at the emergency room.

KEYWORDS: Surgery; Trauma; Ultrasonography; Pneumothorax; Emergency.

\section{INTRODUÇÃO}

Pneumotórax (PTX) é uma situação clínica encontrada com relativa frequência na sala de emergência. Resulta da presença ou acúmulo de ar na cavidade pleural, como consequência da solução de continuidade da integridade das pleuras. Classifica-se em espontâneo (primário ou secundário) e não espontâneo (traumático) (GOMES, 2015, p. 1). 0 PTX traumático (motivo de interesse do presente relato de caso) surge decorrente de trauma de tórax aberto ou fechado (que correspondem a $90 \%$ dos traumas torácicos) (ANNES; MARTINS FILHO, 2015, p. 1), podendo ainda ser resultado de procedimentos intervencionistas com finalidade terapêutica ou diagnóstica.

Rotineiramente pacientes vítimas de traumas são abordadas por meio de exame clínico, somados aos exames de imagens já bem estabelecidos e estudados: raios- $\mathrm{x}(\mathrm{RX})$ e tomografia computadorizada (TC). Mas atualmente 0 uso da EFAST (Extended Focused Assessment with Sonography for Trauma) à beira do leito tem se mostrado superior (em termos de sensibilidade) aos raios- $X$ de tórax e semelhante (em sensibilidade e especificidade) à TC de tórax, exame reconhecido como padrão-ouro na identificação do PTX (ANNES; MARTINS FILHO, 2015, p. 18).

0 uso de US em pacientes vítimas de trauma, segundo 0 protocolo FAST (Avaliação Ultrassonográfica Direcionada para Trauma), é bem difundido em nosso meio. Sua inclusão na avaliação primária do trauma, de acordo com o ATLS (Suporte Avançado de Vida no Trauma), sob o comando do Colégio Americano de Cirurgiões, permitiu avanços significativos no tratamento dos doentes atendidos em salas de emergência e pronto-socorro. (ATLS, 2012). É uma ferramenta que permite rápida avaliação do traumatizado, pode ser repetida várias vezes e, entre outros benefícios, não expõe 0 paciente à radiação ionizante. Exige apenas um aparelho de ultrassom e mínima experiência do examinador.

Classicamente, de acordo com o Colégio Americano de Cirurgiões (2012), a avaliação com FAST é voltada para a investigação de trauma abdominal e do pericárdio, na busca de sinais de líquido livre intracavitário, e examina quatro regiões fundamentais: a) janela pericárdica; b) janela do quadrante superior direito, incluindo a interface fígado-diafragma e 0 espaço de Morrison; c) janela do quadrante superior esquerdo, incluindo as interfaces baço-diafragma $e$ rim-baço; d) janela suprapúbica.

Com 0 objetivo de melhorar a avaliação do paciente, foi desenvolvida uma extensão do protocolo FAST, denominada EFAST ou FASTEstendido, que ampliou a avaliação antes reservada à parede abdominal e cardíaca para a cavidade torácica, possibilitando a detecção de pneumotórax e hemotórax. Segundo Flato et al. (2010), as indicações de EFAST incluem:

- trauma cardíaco penetrante e fechado;

- trauma abdominal fechado;

- trauma torácico;

- pneumotórax;

- hemotórax;

- hipotensão de causa não definida.

Para tanto, avalia:

- recesso hepatorrenal;

- linha axilar direita anterior (transição fígado-pulmão-diafragma);

- linha hemiclavicular anterior direita entre $03^{\circ}$ e 0 $5^{\circ}$ espaço intercostal (avaliação anterior do hemitórax direito);

- recesso esplenorrenal; 
- linha axilar esquerda anterior, transição baço -pulmão;

- linha hemiclavicular anterior esquerda entre $03^{\circ} \mathrm{e}$ $05^{\circ}$ espaço intercostal (avaliação anterior do hemitórax esquerdo); espaço retrovesical (espaço de Douglas);

- janela pericárdica (corte subxifoide 4 câmaras cardíacas).

No exame do tóraxà beira do leito, com paciente em posição supina e usando um transdutor linear de baixa frequência, posicionado longitudinalmente na parede torácica, 0 examinador busca 0 ponto "mais alto" do tórax, onde 0 ar se acumula. Esse ponto geralmente se encontra entre 0 terceiro e 0 quinto espaço intercostal na linha hemiclavicular. As estruturas que devem ser identificadas durante 0 exame são: linha pleural (composta pela pleura visceral e parietal), arcos costais e parênquima pulmonar (FLATO et al., 2010).

0 padrão esperado para US torácico normal recebe o nome de sea over the sand (Figura 1) ou seashoresign, (em nosso meio, "horizonte e areia da praia"). No modo M do US, encontramos um padrão linear no campo próximo ao transdutor e, no campo mais distal, um padrão granular. Quando ocorre perda dessa relação, com padrão linear no campo próximo e no campo mais distal do transdutor, consideramos anormal e diagnóstico de PTX.

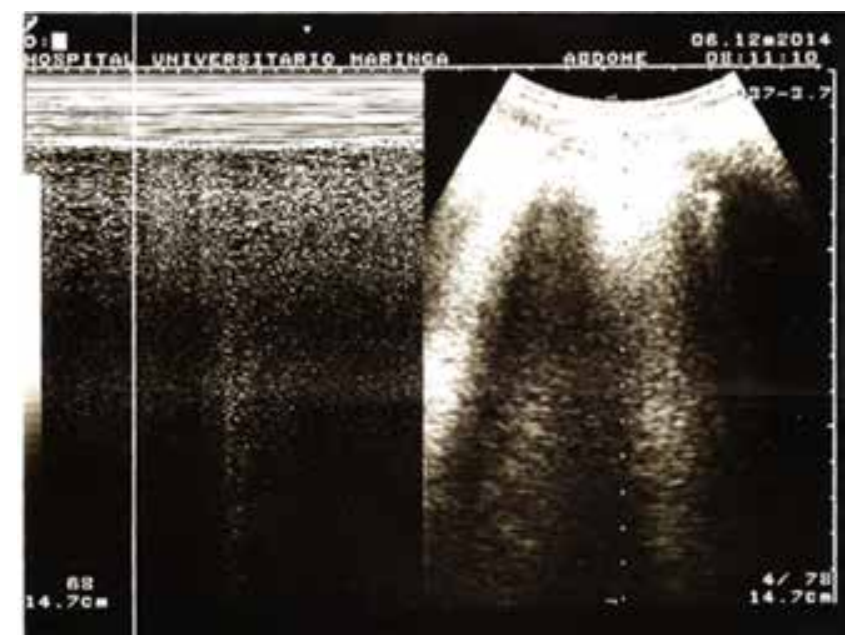

Figura 1. Padrão "sea over the sand" considerado normal no US do tórax

Fonte: Prontuário do Hospital Universitário Regional de Maringá (HURM)
Além de observar 0 padrão geral normal, consideramos também na avaliação ultrassonográfica do tórax: Sliding Sign (deslizamento dos folhetos pleurais), Comet Tails Artifact ou linhas-B e o Lung Point.

Sliding Sing, ou deslizamento pleural, é o movimento dinâmico entre os folhetos parietal e visceral da pleura. Trata-se de uma linha hiperecogênica, logo abaixo dos arcos costais, que em situações normais apresenta um movimento de deslizamento, facilmente perceptível ao exame. Ausência desse sinal indica presença de PTX.

Comet Tails Artifacts, ou linhas-B, são linhas de reflexões hiperecoicas, com origem na pleura visceral e que se dirigem perpendicularmente para 0 parênquima pulmonar. Possuem uma base estreita e movem-se sincronicamente com a respiração. Sua ausência também está relacionada com PTX (SOLDATI et al., 2008).

Para definir a presença ou não do PTX, devemos ainda pesquisar Lung Points. Estes pontos demarcam a transição entre a região onde há deslizamento normal dos folhetos pleurais (o SlidingSign) e a região onde não existe deslizamento. 0 escaneamento ultrassonográfico dos dois pulmões deve ser realizado no sentido ântero-lateral-posterior, em inspiração profunda. Com esse ponto, podemos determinar a extensão e a correta localização do PTX (quanto mais lateral o LungPoint, maior o PTX). Segundo Ball e colaboradores (2005), isso tem importante correlação clínica, pois 0 tamanho e a distribuição do PTX e 0 volume de ar intrapleural relacionam-se com 0 tamanho do escape de ar e com o tempo necessário para a correta resolução do quadro.

A localização do PTX está relacionada diretamente com os efeitos da gravidade, a elasticidade pulmonar e a da parede torácica e a relação entre o pulmão com as estruturas hilares e 0 ligamento pulmonar inferior. Puyana e colaboradores (2008) classificaram em apical (superior ao manúbrio ou ao primeiro arco costal), basal (próximo, ou tocando 0 diafragma), anterior/medial (passando pelo plano coronal medialmente ao parênquima pulmonar), 
lateral (passando pelo plano coronal lateralmente ao parênquima pulmonar) ou posterior.

Portanto, 0 objetivo deste estudo é de apresentar um relato de caso sobre a utilização da EFAST na sala de emergência no diagnóstico de pneumotórax, evidenciando sua fácil aplicabilidade e os benefícios que traz para o paciente.

\section{METODOLOGIA}

Trata-se de um relato de caso, que foi conduzido no Hospital Universitário Regional de Maringá (HURM), no período do mês de novembro do ano de 2014, considerando todo o quadro clínico do paciente, desde sua admissão hospitalar até sua alta. Utilizamos referência bibliográfica atual (livros, artigos, revistas científicas, anais de congressos), disponível em meios físico e virtual, em plataformas de pesquisas online. Foram usadas, entre outras, algumas palavras-chave para a pesquisa, como "pneumotórax", "trauma", "emergência", "ATLS", "FAST". Não foi realizada exclusão de publicações por data, mas preferimos bibliografias mais atualizadas. 0 trabalho conta com consentimento legal do paciente.

\section{RELATO DO CASO}

Paciente do sexo masculino, de 65 anos, admitido no Hospital Universitário Regional de Maringá (HURM), vítima de colisão automobilística, (condutor do veículo, usando cinto de segurança e em baixa velocidade - SIC) dois dias antes da admissão. Deu entrada com quadro de dor em região lombar, de moderada intensidade, aumento gradativo e piora com a movimentação. Dor torácica moderada, tipo pontada, ventilatório-dependente, mais intensa no hemitórax esquerdo, sem irradiação, sem melhora significativa com analgesia convencional. Associados, referia também dispneia aos grandes esforços, desconforto respiratório pior nas últimas $24 \mathrm{~h}$, mas sem ortopneia e sem dispneia paroxística noturna. Negava febre, negava tosse, negava cefaleia. Não apresentou alterações de nível de consciência, decorrentes do trauma, nem mesmo síncope ou alterações sensitivomotoras. Como comorbidades, apresenta HAS, em uso de medicação (não sabe referir o nome). Sem história de cirurgias prévias.

Foi atendido inicialmente em sala de emergência, encontrava-se em bom estado geral, lúcido e orientado têmporo-espacialmente, anictérico, acianótico e afebril, dispneico (22irm), hipertenso (206x123mmHg), 84bpm, saturação de 0, 96\% em ar ambiente.

Ausculta pulmonar com murmúrio vesicular presente, diminuído no $1 / 3$ inferior do hemitórax à esquerda, sem ruídos adventícios e frequência respiratória de 22irm. Ausculta cardíaca apresentava bulhas cardíacas rítmicas, normofonéticas, em dois tempos e sem sopros. Abdome apresentava-se normotenso, com ruídos hidroaéreos presentes e normoativos, sem visceromegalias, sem dor à palpação, sem sinais de irritação peritoneal. Membros superiores e inferiores sem alterações, pulsos periféricos presentes, palpáveis, simétricos, cheios e regulares. Escoriações na face anterior do hemitórax esquerdo com dor à palpação. Sem enfisema subcutâneo.

Foi submetido a E-FAST (com transdutor convexo de $3,5 \mathrm{MHz}$ ), à beira do leito, que evidenciou pneumotórax simples à esquerda (Figura 2), confirmado em $\mathrm{RX}$ realizado no leito, na sala de emergência. Outras regiões investigadas não apresentavam alterações. Prontamente submetido à toracostomia com drenagem pleural fechada à esquerda, com dreno de tórax de número 36 , conectado em sistema de selo d'água. Procedimento realizado de acordo com as normas e rotinas do serviço de cirurgia torácica do HURM, sem intercorrências, com drenagem efetiva do ar em espaço pleural. 


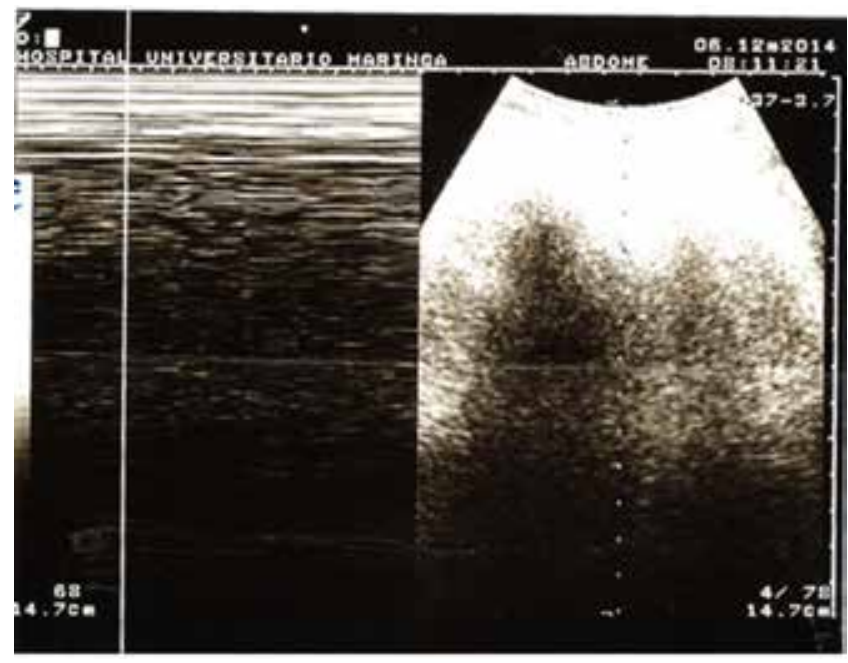

Figura 2. Pneumotórax

Fonte: Prontuário do Hospital Universitário Regional de Maringá (HURM)

Após estabilização do quadro, melhora do padrão ventilatório e da dor apresentada, foi transferido para enfermaria de clínica cirúrgica, onde recebeu tratamento com dieta, analgesia, hidratação e medicamentos, segundo rotina do serviço. Exames laboratoriais de internamento não mostraram alterações significativas, bem como não apresentou febre, alterações de hábitos fisiológicos ou de qualquer outro sistema. Evoluiu de maneira satisfatória, com melhora do padrão respiratório e da dor torácica. Evolução radiológica também adequada, com reexpansão pulmonar, diminuição do débito da drenagem e sem escape aéreo. Recebeu alta no quarto dia de internamento, depois da retirada do dreno torácico e melhora clínica.

\section{DISCUSSÃO}

0 uso do US no trauma em seres humanos foi registrado pela primeira vez com Wernecke et al. (1987). Na América do Norte, no início da década de 1990, centros de traumas realizaram estudos que comparavam US, TC e lavado peritoneal (LP) a fim de avaliar sua utilidade diagnóstica (PUYANA et al., 2008). Desde então foi ganhando abrangência mundial e importância no manejo de pacientes traumatizados. Ao contrário dos países desenvolvidos, na América
Latina ainda são pobres os estudos, e seguem-se os protocolos das instituições internacionais, como 0 ATLS e o Colégio Americano de Cirurgiões.

Estudos norte-americanos e europeus empenham-se em demonstrar, estatisticamente, as diferenças entre os principais meios diagnósticos em trauma: RX, TC e US. Embora extremamente importantes no exame complementar em trauma (sobretudo no Brasil), tanto RX quanto TC têm limitações. $0 \mathrm{RX}$ anteroposterior de tórax é um método de imagem bastante acessível, de relativo baixo custo e que pode ser interpretado pelo médico assistente, seja qual for sua especialidade, com certa segurança. Mas, comparado ao US, apresenta sensibilidade na detecção de PTX que varia de 28 a 75\% (WILKERSON; STONE, 2010) e, mesmo com especificidade próxima a 100\%, tem limitações (exemplo, incidência alta de falha no diagnóstico de PTX oculto). A TCT, considerada padrão-ouro no diagnóstico do PTX, tem vantagens claras em relação ao RXT anteroposterior. (PUYANA et al., 2008). Entretanto, tem restrições: alto custo, baixa disponibilidade, tempo prolongado entre admissão do paciente-execução-interpretação, exposição do doente às altas doses de radiação e por vezes depende de médico especialista para interpretá-la.

Atualmente, com 0 conhecimento das alterações produzidas pelas doenças intersticiais, PTX e atelectasias, além de ser empregado para 0 estudo de estruturas pleurais e doenças adjacentes, seu uso foi ampliado para situações de trauma e medicina intensiva. É um exame facilmente realizado à beira do leito, tem boa portabilidade, de rápida execução, não expõe paciente à radiação e tem baixo custo. Se executado de maneira correta e conhecendo seus pontos principais, podemos, com sensibilidade de 86\% e especificidade de 97\% (ZHANG et al., 2006), diagnosticar PTX na sala de emergência e promover rápido tratamento ao nosso doente. A presença isolada de Sliding Sign exclui o diagnóstico de PTX com alta sensibilidade, especificidade, valor preditivo negativo e valor preditivo positivo. Ausência de Comet Tails Artifacts isolada confirma PTX com alta acurácia e valor preditivo negativo. Quando temos a presença 
destes dois marcadores, podemos excluir, com grande acurácia, a presença de PTX. Do mesmo modo, a identificação do Lung Point permite definir, com grande segurança, o tamanho e a localização do PTX.

De maneira geral podemos entender o EFAST na Figura 3.

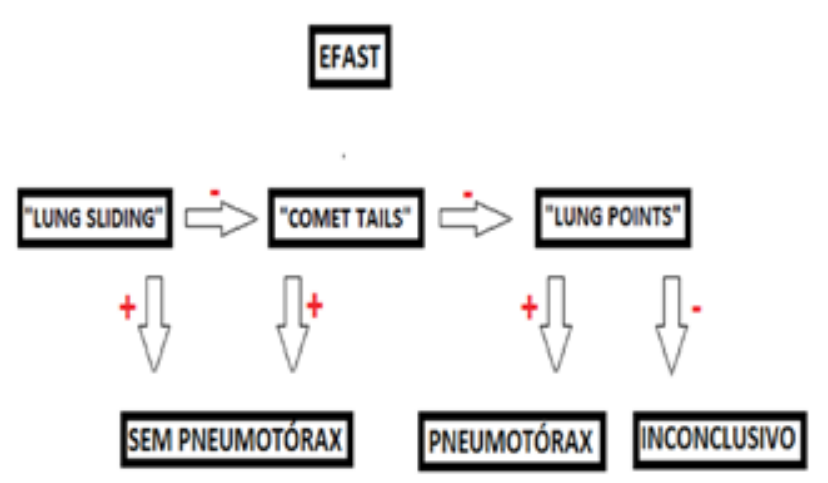

Figura 3. EFAST - Extended Focused Assessment With Sonography for Trauma.

Fonte: Acervo dos autores (2015).

Apesar de todos os benefícios, como simplicidade, segurança e portabilidade, cabe ressaltar que 0 US de tórax tem limitações no diagnóstico de PTX, como ocorre quando há presença de enfisema subcutâneo, adesões e calcificações pleurais ou traumas de pele.

0 tratamento de pacientes com PTX é bastante variável, desde repouso e observação, oxigenoterapia suplementar, aspiração simples, drenagem pleural fechada, videotoracoscopia ou toracotomia aberta com abordagem de bolhas, abrasão pleural e pleurectomia. A escolha da melhor opção dependerá de fatores como a intensidade dos sintomas e repercussão clínica, magnitude, etiologia, comorbidades pleurais associadas, doença pulmonar subjacente e persistência ou recorrência do pneumotórax. 0s principais objetivos são: livrar o espaço pleural do ar contido, restabelecer a função pulmonare diminuir a probabilidade de recorrência.

\section{CONCLUSÃO}

Vítimas de trauma respondem por grande parte dos atendimentos em prontos-socorros. Dentro desse contexto, disponibilizar o US na sala de emergência propicia ao médico assistente uma ferramenta de extrema importância no diagnóstico e na correta condução desses pacientes. $E$ isso se deve à diminuição no tempo entre a admissão e 0 tratamento definitivo do traumatizado, fato que, não raro, é definidor de sobrevida.

0 conhecimento sobre EFAST, oferecendo aparelhos e treinamento básico para os médicos assistentes, mudaria de modo muito positivo o perfil dos nossos hospitais. Dentro de nossa instituição podemos perceber, ainda que não existam registros ou trabalhos específicos, evolução quanto ao atendimento do traumatizado com a introdução dessa nova metodologia de exame de imagem. Esse aprendizado pode ser adquirido com a simples observação de um exame realizado em mãos experientes e sendo reproduzido posteriormente. Mas também ganharia muito em qualidade com o estabelecimento de normas e protocolos rígidos, introduzindo o uso do EFAST na rotina do atendimento ao politraumatizado.

0 Hospital Universitário Regional de Maringá oferece 0 equipamento satisfatório para realização do exame, ambiente físico adequado e possibilidades de resolução dos quadros que são apresentados. Ainda assim, existe espaço para melhoria e evolução do atendimento. Notamos que 0 treinamento de maior número de médicos assistentes, incentivandoos com programas didáticos, traria importante contribuição. Talvez o treinamento técnico seja a principal limitação do emprego da EFAST em nosso meio, pois, para o ambiente hospitalar brasileiro, ainda é tema relativamente novo, carente de mais fontes bibliográficas.

Estimular seu amplo uso significa diminuir tempo entre admissão e tratamento do paciente, aumentando a sobrevida deste. Representa também diminuição de custos, de exposição à radicação e não deixa de ser estímulo ao aprendizado e à atualização. 
Frente à realidade da saúde nacional, mostra-se uma solução bastante plausível, que pode ser realizada, trazendo grande contribuição social.

\section{REFERÊNCIAS}

ANNES, R.; MARTINS FILHO, S. C. Trauma de Tórax Fechado. In: SOCIEDADE Brasileira de Cirurgia Torácica. Tópicos de atualização em cirurgia torácica. Disponível em: <http://itarget.com.br/newclients/ sbct/wp-content/uploads/2015/03/trauma_torax_ fechado.pdf > . Acesso em: 9 mar. 2016.

BALL, C. G. et al. Factors related to the failure of radiographic recognition of occult posttraumatic pneumothoraces. The American Journal of Surgery, Birmingham, AL, v. 189, n. 5, p. 541-546, maio 2005. Disponívelem:<http://www.americanjournalofsurgery. com/article/S0002-9610(05)00146-7/pdf>. Acesso em: 10 mar. 2016.

COLÉGIO AMERICANO DE CIRURGIÕES. Comitê de Trauma. ATLS: Advanced Trauma Life Support (Suporte Avançado de Vida no Trauma). Manual do Curso de Alunos. 9. ed. Chicago, 2012. Disponível em: <http:// pt.slideshare.net/matheusgaldino355/atls-9ed>. Acesso em: 9 mar. 2016.

FLATO, U. A. P. et al. Utilização do FAST-Estendido (EFAST - Extended Focused Assessment with Sonography for Trauma) em terapia intensiva. Revista Brasileira de Terapia Intensiva, v. 22, n. 3, p. 291299, 2010. Disponível em: <http://www.scielo.br/pdf/ rbti/v22n3/12.pdf>. Acesso em: 10 mar. 2016.

GOMES, C. A. Pneumotórax. In: SOCIEDADE Brasileira de Cirurgia Torácica. Tópicos de atualização em cirurgia torácica. Disponível em: <http://itarget.com. br/newclients/sbct/wp-content/uploads/2015/03/ pneumotorax.pdf > . Acesso em: 9 mar. 2016.

PRINA, E.; TORRES, A.; CARVALHO, C. R. R. Ultrassom de pulmão na avaliação de derrame pleural. Jornal
Brasileiro de Pneumologia, v. 40, n. 1, p. 1-5, 2014. Disponível em: <http://www.jornaldepneumologia. com.br/detalhe_artigo.asp?id=2242>. Acesso em: 9 mar. 2016.

PUYANA, J. C. et al. Ultra-som em emergências e trauma - USET. Bogotá: Distribuna Ed. y Librería Médica, 2008. Disponível em: <http://www. panamtrauma.org/Resources/Distribuna/Puyana $\% 20$ \%20USET\%20portugues.pdf>. Acesso em: 10 mar. 2016.

WERNECKE, K. et al. Pneumothorax: evaluation by ultrasound - preliminary results. Journal of Thoracic Imaging, Boston, MA, v. 2, n. 2, p. 76-78, 1987. Disponível em: <http://www.ncbi.nlm.nih.gov/ pubmed/3298684>. Acesso em: 10 mar. 2016.

WILKERSON, R. G.; STONE, M. B. Sensitivity of bedside ultrasound and supine anteroposterior chest radiographs for the identification of pneumothorax after blunt trauma. AcademicEmergency Medicine, Malden, MA, v. 17, n. 1, p. 11-17, jan. 2010. Disponível em: <http://onlinelibrary.wiley.com/doi/10.1111/ j.1553-2712.2009.00628.x/abstract>. Acesso em: 10 mar. 2016.

ZHANG, M. et al. Rapid detection of pneumothorax by ultrasonography in patients with multiple trauma. Critical Care, London, v. 10, n. 4, R 112, ago. 2006. Disponível em: <http://www.ncbi.nlm.nih.gov/pmc/ articles/PMC1751015/>. Acesso em: 10 mar. 2016.

Recebido em:20 de março de 2016 Aceito em: 29 de abril de 2016 